\title{
Equação de volume para espécies comerciais em Floresta Ombrófila Densa no sul de Roraima
}

\author{
Helio Tonini ${ }^{1 *}$, Renato Alves Borges ${ }^{2}$ \\ ${ }^{1}$ Embrapa Agrossilvipastoril. Rodovia MT 222, CEP 78550-970, Sinop, MT, Brasil \\ ${ }^{2}$ Secretaria de Agricultura, Pecuária e Agronegócio de Roraima. Avenida Galycon de Paiva 1957, Centro, CEP 69303-340, Boa Vista, RR, Brasil
}

\author{
"Autor correspondente: \\ helio.tonini@embrapa.br \\ Termos para indexação: \\ Cubagem rigorosa \\ Amazônia \\ Volumetria \\ Manejo florestal
}

Index terms:

Wood volume determination

Amazônia

Volumetry

Forest Management

Histórico do artigo:

Recebido em 24/06/2014

Aprovado em 29/04/2015

Publicado em 30/06/2015

doi: 10.4336/2015.pfb.35.82.738

\begin{abstract}
Resumo - Este trabalho teve como objetivo ajustar e selecionar modelos matemáticos para a estimativa do volume comercial com casca para espécies comerciais de uma Floresta Ombrófila Densa de Terra Firme localizada no sul de Roraima. Foram abatidas e cubadas pelo método de Smalian 122 árvores de 18 espécies com diâmetro à altura do peito maior do que $50 \mathrm{~cm}$. Foram testados nove modelos, ajustados pelos métodos dos mínimos quadrados, e selecionados em função do coeficiente de determinação ajustado, do erro padrão de estimativa em porcentagem, do valor de $\mathrm{F}$ calculado e da análise gráfica da distribuição dos resíduos. Recomendou-se o modelo de Schumacher-Hall. O modelo de Kopezki-Gherardt pode ser empregado, quando não se utilizar dados de altura comercial.
\end{abstract}

\section{Volume equation for commercial species in tropical rain forest in southern Roraima}

\section{Introdução}

A necessidade de quantificação do estoque de matéria prima florestal e a busca pela máxima produção e rentabilidade da floresta justificam a busca por ferramentas que possibilitem estimativas precisas do volume comercial das espécies Amazônicas (Thaines et al., 2010). As equações de volume são de uso geral e indispensáveis para o planejamento e a execução de um plano de manejo florestal, sendo necessário ajustá-las para diferentes espécies, regiões e tipos fisionômicos.
Alguns autores têm disponibilizado equações de volume para diferentes regiões da Amazônia (Fernandes et al., 1983; Silva \& Araujo, 1984 Higuchi \& Ramm, 1985; Rolim et al., 2006; Barros \& Silva Junior, 2009; Colpini et al., 2009; Thaines et al., 2010), porém, devido às grandes dimensões e à diversidade fisionômica e estrutural das florestas na região, tais estudos ainda são considerados insuficientes, sendo necessário abranger uma maior número de espécies, regiões e tipos florestais. 
O ajuste de modelos volumétricos desenvolvidos especificamente para as áreas de cada plano de manejo florestal constitui o procedimento mais eficiente, econômico e preciso para a quantificação do volume de uma floresta (Barros \& Silva Junior, 2009). Por esse motivo, as estimativas por meio de equações de volume tornaram-se obrigatórias e prevista em lei a partir de 2002 (Brasil, 2003).

No entanto, a utilização generalizada da fórmula alemã $\mathrm{v}=\mathrm{g}$.h.f para o cálculo do volume individual com a utilização do fator de forma proposto por Heinsdjik \& Bastos (1963) de 0,7 para todas as espécies, regiões e tipologias florestais da Amazônia ainda é aceita e muito utilizada pelos técnicos na região. Segundo Rolim et al. (2006), este tipo de estimativa é menos precisa e subestima o volume real, quando comparado com os valores obtidos a partir de modelos volumétricos desenvolvidos localmente.

Este trabalho teve como objetivo ajustar e selecionar modelos matemáticos para a estimativa do volume comercial com casca para espécies comerciais de uma Floresta Ombrófila Densa de Terra Firme localizada no sul de Roraima.

\section{Material e métodos}

\section{Caracterização da área do estudo}

Os dados foram coletados em um Plano de Manejo Florestal Sustentado pertencente à Empresa Madeireira Vale Verde LTDA, localizado em uma Floresta Ombrófila Densa de Terra Firme, no município de Caracaraí na região sul do estado de Roraima South American Datum 1969; Zona 20N; Coordenadas UTM: E 729975 e N 203744.

O clima, segundo a classificação Köppen é do tipo Am com precipitação entre 1.700 a $2.000 \mathrm{~mm}$ anuais (Barbosa, 1997). A topografia da área é suave à ondulada, com altitude variando de 60 a 120 m na parte oeste e uma região de serra na parte sudoeste, onde a altitude pode chegar a $330 \mathrm{~m}$. O solo predominante na área é o Argissolo Vermelho-Amarelo (Vale Verde, 2012).

Segundo Condé \& Tonini (2013), a comunidade florestal pode ser considerada bem estruturada, madura e diversa, em bom estado de conservação. As famílias com maior número de indivíduos são Fabaceae, Lecythidaceae e Sapotaceae. Segundo os autores, o índice de diversidade de Shannon $\left(\mathrm{H}^{\prime}=3,27\right)$ e o valor de equabilidade de Pielou $(\mathrm{J}=0,64)$ são um pouco inferiores aos obtidos em outros inventários florísticos na Amazônia Legal.

As espécies Pentaclethra macroloba, Eschweilera bracteosa e Pouteria caimito apresentaram os maiores valores de importância. Considerando um diâmetro limite de levantamento de $10 \mathrm{~cm}$, foi observada uma média de 525 árvores.ha-1, $24,6 \mathrm{~m}^{2}$.ha ${ }^{-1}$ de área basal e $521,5 \mathrm{~m}^{3}$.ha ${ }^{-1}$ de volume total. $\mathrm{O}$ volume comercial, considerando espécies comerciais com diâmetro $\geq 50 \mathrm{~cm}$, é de 79,45 $\mathrm{m}^{3} \mathrm{ha}^{-1}$ (Condé \& Tonini, 2013).

\section{Cubagem rigorosa dos indivíduos}

A seleção das espécies comerciais foi realizada com base nos critérios da empresa. Foram abatidas e cubadas pelo método de Smalian, 122 árvores de 18 espécies com diâmetro à altura do peito (DAP) maior do que $50 \mathrm{~cm}$ (Tabela 1). Cada árvore amostra foi identificada pelo nome comum, determinado o seu DAP, a altura comercial (diâmetro a um determinado ponto no tronco onde galhos, defeitos ou tortuosidades inviabilizam o uso em madeira serrada) e calculado o volume comercial com casca através da medição do diâmetro com casca na base, a $0,7 \mathrm{~m}$, a 1,30 $\mathrm{m}$ e a partir desta posição a cada metro do fuste comercial.

O nome científico foi obtido com base na lista de espécies comerciais da empresa, que por sua vez foi obtida após a coleta de material botânico e a retirada de discos de madeira para a identificação botânica e anatômica, realizados no Instituto Nacional de Pesquisa da Amazônia (INPA).

\section{Modelos testados e critérios de seleção}

Os modelos testados (Tabela 1) são os mais comumente utilizados em trabalhos científicos de mesma natureza e foram ajustados pelos métodos dos mínimos quadrados (Draper \& Smith, 1998). Para a seleção do melhor modelo, considerou-se o coeficiente de determinação ajustado (1); o erro padrão de estimativa em porcentagem (3), que foi recalculado no caso de equações logarítmicas (5), conforme Bartoszeck et al. (2002); o fator de inflação da variância (6), rejeitando-se modelos com valor de VIF maiores do que 10; o valor e significância de F de Ficher-Snedecor para o modelo; a significância dos coeficientes pela análise do valor e da significância de $\mathrm{t}$ de Student; e a análise gráfica dos resíduos.

$$
\mathrm{R}_{\mathrm{aj}}^{2}=\mathrm{R}^{2}-\left(\frac{\mathrm{K}-1}{\mathrm{~N}-\mathrm{K}}\right) \mathrm{X}\left(1-\mathrm{R}^{2}\right)
$$


Syx $=\sqrt{\text { QMresíduo }}$

$\operatorname{Syx} \%=\frac{S y x}{-} * 100$

y

$S y x_{\text {recalculado }}=\sqrt{\frac{\sum(\hat{y}-y)}{n-p}}$

Syx $x_{\text {recalculado }} \%=\frac{S y x_{\text {recalculado }}}{-}$

$y$

$$
V I F=\frac{1}{1-R^{2}}
$$

Onde: $\mathrm{K}$ = número de variáveis independentes da equação; $\mathrm{N}$ = número de observações; $\mathrm{R}^{2}$ = coeficiente de determinação; Syx = erro padrão da estimativa; QMresíduo = quadrado médio do resíduo obtido na análise de variância; $\hat{y}$ = volume estimado pela equação; $\mathrm{y}=$ volume observado; $\mathrm{n}=$ número de observações; $\mathrm{p}=$ número de coeficientes; $\bar{y}=$ média aritmética do volume comercial

Tabela 1. Modelos matemáticos ajustados para estimar o volume comercial com casca em uma Floresta Ombrófila Densa de Terra firme em Caracarai, RR.

\begin{tabular}{cll}
\hline Número & \multicolumn{1}{c}{ Modelo } & \multicolumn{1}{c}{ Autor } \\
\hline $\mathbf{1}$ & $V=b_{0}+b_{1} d$ & Berkout \\
$\mathbf{3}$ & $V=b_{0}+b_{1} d+b_{2} d^{2}$ & Hohenaldl-Kren \\
$\mathbf{4}$ & $\mathrm{h} V=b_{0}+b_{1} \mathrm{~h} d+b_{2} 1 / d$ & Brenac \\
$\mathbf{5}$ & $V=b_{0}+b_{1} \mathrm{~h} d$ & Kopezki-Gherardt \\
$\mathbf{6}$ & $V=b_{1} d^{2}+b_{2} d^{2}$ & Dissescu-Meyer \\
$\mathbf{7}$ & $\mathrm{h} V=b_{0}+b_{1} \mathrm{~h} d^{2} h$ & Spurr \\
$\mathbf{8}$ & $\mathrm{h} V=b_{0}+b_{1} \mathrm{~h} d+b_{2} \mathrm{~h} h$ & Schumacher-Hall \\
$\mathbf{9}$ & $V=b_{0}+b_{1} d^{2}+b_{2} d^{2} h c+b_{3} d h c^{2}+b_{4} h c$ & Naslund \\
\hline
\end{tabular}

Onde: $\mathrm{V}=$ volume comercial com casca individual $\left(\mathrm{m}^{3}\right) ; \mathrm{d}=$ diâmetro à altura do peito $(\mathrm{cm}) ; \mathrm{hc}=$ altura comercial $(\mathrm{m}) ; \mathrm{B}_{\mathrm{j}}=$ coeficientes dos modelos. 


\section{Resultados e discussão}

O volume comercial com casca individual variou entre $4,28 \mathrm{~m}^{3}$ e $12,94 \mathrm{~m}^{3}$, sendo o menor observado para Qualea acuminata e o maior para Ocotea caudata (Tabela 2). A altura comercial média foi de 15,2 m, sendo a maior média observada para as árvores de Buchenavia oxicarpa, com 17,3 $\pm 3,4 \mathrm{~m}$, e a menor para as de Piptadenia suavolens, com 12,1 $\mathrm{m} \pm 3,7 \mathrm{~m}$. $\mathrm{O}$ fator de forma comercial médio foi de 0,83 , variando de 0,69 a 0,93 .

Tabela 2. Número de árvores amostradas para o ajuste dos modelos volumétricos (N), médias e devio padrão (s) por espécie de diâmetro do fuste tomado a 1,30 m do solo (DAP), altura comercial (Hc), volume comercial com casca $\left(\mathrm{VC}_{\mathrm{cc}}\right)$ e fator de forma comercial (fc).

\begin{tabular}{lcccccccccc}
\hline \multicolumn{1}{c}{ Espécie } & Nome comum & $\mathbf{N}$ & $\begin{array}{c}\mathbf{D A P} \\
\mathbf{( c m )}\end{array}$ & $\mathbf{s}_{\mathbf{D A P}}$ & $\begin{array}{c}\mathbf{H c} \\
\mathbf{( m )}\end{array}$ & $\mathbf{s}_{\mathbf{h c}}$ & $\begin{array}{c}\mathbf{V C}_{\mathbf{c c}} \\
\left(\mathbf{m}^{3}\right)\end{array}$ & $\mathbf{s}_{\mathbf{v c c c}}$ & $\mathbf{f c}$ & $\mathbf{s}_{\mathbf{f c}}$ \\
\hline Vochysia guianensis & Amarelão & 1 & 84,0 & - & 15,3 & - & 6,42 & - & 0,76 & - \\
Vataireopsis speciosa & Amargoso & 5 & 71,5 & 11,2 & 16,9 & 2,6 & 5,11 & 2,34 & 0,71 & 0,02 \\
Dinizia excelsa & Angelim Ferro & 11 & 105,5 & 24,3 & 15,8 & 2,5 & 11,58 & 5,12 & 0,81 & 0,06 \\
Hymenolobium complica- & Angelim Pedra & 5 & 83,5 & 6,1 & 16,1 & 2,6 & 7,71 & 1,62 & 0,88 & 0,06 \\
tum & & & & & & & & & & \\
Ocotea caudata & Breu Manga & 2 & 101,9 & 34,4 & 16,3 & 1,4 & 12,94 & 8,43 & 0,88 & 0,09 \\
Erisma uncinatum & Caferana & 5 & 67,3 & 7,2 & 14,9 & 2,6 & 4,71 & 1,35 & 0,78 & 0,06 \\
Anacardium giganteum & Cajuí & 3 & 88,9 & 8,4 & 16,6 & 2,3 & 8,07 & 1,81 & 0,69 & 0,11 \\
Vochysia máxima & Cedrinho & 1 & 117,6 & - & 15,3 & - & 11,43 & - & 0,83 & - \\
Goupia glabra & Cupiuba & 16 & 73,3 & 8,8 & 13,7 & 2,3 & 4,85 & 1,64 & 0,93 & 0,07 \\
Couratari multiflora & Estopeiro & 2 & 87,5 & 25,4 & 16,3 & 7,1 & 8,43 & 2,07 & 0,88 & 0,09 \\
Parkia paraensis & Faveira vermelha & 2 & 87,8 & 7,8 & 15,3 & 0 & 8,22 & 1,56 & 0,83 & 0,01 \\
Buchenavia oxicarpa & Mirindiba & 2 & 66,7 & 11,3 & 17,3 & 3,4 & 4,64 & 1,81 & 0,80 & 0,07 \\
Pouteria speciosa & Pajura & 6 & 77,6 & 5,9 & 13,0 & 2,8 & 5,26 & 0,24 & 0,81 & 0,03 \\
Caryocar glabrum & Piquiá & 8 & 100,6 & 9,7 & 12,8 & 3,8 & 8,37 & 2,28 & 0,88 & 0,11 \\
Qualea acuminata & Quaruba & 4 & 63,3 & 18,2 & 16,8 & 1,4 & 4,28 & 3,16 & 0,87 & 0,08 \\
Piptadenia suavolens & Timborana & 5 & 75,9 & 7,0 & 12,1 & 3,7 & 4,76 & 1,24 & 0,76 & 0,01 \\
\hline
\end{tabular}

As variações individuais de volume comercial com casca estão dentro da faixa de 2,4 a 21,80 $\mathrm{m}^{3}$ observado por Rolim et al. (2006); Colpini et al. (2009) e Thaines et al. (2010). O fator de forma artificial médio com casca foi mais elevado do que sugerido por Heinsdjik \& Bastos (1963), Nogueira et al. (2008), Colpini et al. (2009) e Thaines et al. (2010), cujos resultados apresentados foram entre 0,70 e 0,78 .

As diferenças podem estar relacionadas ao emprego de diferentes alturas e diâmetros no cálculo do volume comercial individual; nas irregularidades no fuste que variam com a espécie; na presença de ocos no tronco e/ ou pelo diâmetro mínimo de amostragem.
O conceito de diâmetro mínimo de utilização é dinâmico e as dimensões regionais podem se alterar de acordo com as especificações da indústria, dependendo do equipamento disponível (Machado \& Figueiredo Filho, 2003). Alguns estudos na Amazônia, como o de Colpini et al. (2009), consideraram a altura do fuste como altura comercial, o que influencia diretamente o cálculo do fator de forma artificial, pois diminui com o aumento da altura, mesmo que duas árvores apresentem a mesma forma geométrica (Finger, 1992).

Segundo Nogueira et al. (2008), as irregularidades no fuste, a presença de troncos ocos e o diâmetro de amostragem exercem uma forte influência sobre a 
estimativa do fator de forma médio de uma floresta. Apesar das árvores grandes estocarem uma grande porção da biomassa, têm pouca influência sobre o fator de forma médio na floresta, que é mais influenciado pelo grande número de árvores pequenas. Segundo os autores, o uso de um fator de forma médio sem a ponderação pelo volume proporcional das árvores maiores resulta em erros nas estimativas de volume por hectare.

Todos os modelos testados foram significativos ao nível de $1 \%$ de probabilidade (Tabela 3). Os valores de $\mathrm{r}^{2}{ }_{\mathrm{aj}}$ e Syx $\%$ variaram entre 0,79 e 0,98 e $10,05 \%$ e $19,78 \%$, respectivamente. Observou-se superioridade para os modelos de dupla entrada, que empregaram a altura comercial como variável independente. Nestes, a variação de $r^{2}{ }_{a j}$ e Syx \% foi de 0,95 a 0,98 e 10,05\% a $10,62 \%$, respectivamente, contra 0,79 a 0,96 e $18,9 \%$ a $19,78 \%$ obtidos para os modelos de simples entrada.

Segundo Rolim et al. (2006), a menor precisão de modelos de simples entrada é esperada, na medida em que estes modelos assumem que árvores de mesmo diâmetro possuem a mesma altura, o que não é verdadeiro para florestas heterogêneas. Esta afirmativa tem sido corroborada por estudos como os de Silva \& Araujo (1984); Barros \& Silva Junior (2009); Thaines et al. (2010), dentre outros; que obtiveram melhores ajustes quando empregaram a altura comercial como variável independente.

Os valores de $\mathrm{r}^{2}{ }_{\mathrm{aj}}$ e Syx\% obtidos neste estudo para os modelos de dupla entrada estão próximos aos observados por Silva \& Araujo (1984); Barros \& Silva Junior (2009) com variações entre 0,71 a 0,99 e $4 \%$ a $18 \%$, respectivamente.

Entre os modelos de dupla entrada, o de Schumacher e Hall apresentou o melhor ajuste e distribuição dos resíduos (Figura 2B), O modelo de Spurr tendeu a subestimar os volumes nas maiores árvores (Figura 2A). Os modelos de Hohenaldl-Kren, Naslund e Brenac apresentaram alta multicolinearidade e coeficientes não significativos.

Corroborando com o presente estudo, a equação de Schumacher e Hall tem sido selecionada para diferentes regiões da Amazônia nos estados do Pará, Mato Grosso e Amazonas (Silva \& Araujo, 1984; Higuchi \& Ramm, 1995; Rolim et al., 2006; Colpini et al., 2009; Thaines et al., 2010).

Tabela 3. Coeficientes e ajuste dos modelos volumétricos.

\begin{tabular}{|c|c|c|c|c|c|c|c|c|c|}
\hline \multirow[t]{2}{*}{ Modelo } & \multicolumn{5}{|c|}{ Coeficientes } & \multicolumn{4}{|c|}{ Ajuste } \\
\hline & $\mathbf{B}_{0}$ & $\mathbf{B}_{1}$ & $\mathbf{B}_{2}$ & $\mathbf{B}_{3}$ & $\mathrm{~B}_{4}$ & $\mathbf{R}_{\text {aj }}^{2}$ & Syx\% & Fcalc & VIF \\
\hline 1 & $-6,86168$ & 0,168331 & - & - & - & 0,83 & 19,78 & 593,36 & 1,0 \\
\hline 2 & $-0,09712^{\mathrm{ns}}$ & $0,013697^{\mathrm{ns}}$ & 0,000838 & - & - & 0,84 & 19,04 & 327,27 & 52,6 \\
\hline 3 & $-5,61712^{\mathrm{ns}}$ & 1,7397 & $-13,5806^{\mathrm{ns}}$ & - & - & 0,79 & 19,13 & 218,74 & 56,4 \\
\hline 4 & $-6,50378$ & 1,902534 & - & - & - & 0,89 & 19,03 & 440,89 & 1,0 \\
\hline 5 & 0,51168 & 0,000911 & - & - & - & 0,84 & 18,90 & 655,48 & 1,0 \\
\hline 6 & - & 0,011546 & 0,00085 & - & - & 0,96 & 18,90 & 2026,7 & 18,4 \\
\hline 7 & $-8,92345$ & 0,937295 & - & - & - & 0,98 & 10,62 & 2527,7 & 1,0 \\
\hline 8 & $-8,89142$ & 1,937052 & 0,824841 & - & - & 0,96 & 10,10 & 1386,9 & 1,0 \\
\hline 9 & $-1,35351^{\mathrm{ns}}$ & 0,000401 & $3,52 \mathrm{E}-05$ & $6,61 \mathrm{E}-07^{\mathrm{ns}}$ & $0,107965^{\text {ns }}$ & 0,95 & 10,05 & 660,97 & 48,2 \\
\hline
\end{tabular}

Onde: $\mathrm{B}_{\mathrm{j}}=$ coeficientes dos modelos; $\mathrm{R}_{\mathrm{aj}}^{2}=$ coeficiente de determinação ajustado; Syx $\%=$ Erro padrão de estimativa em porcentagem; $\mathrm{F}$ calc $=$ valor de F calculado; VIF = Fator de inflação da variância; ns = coeficiente não significativo a 5\% pelo teste t de Student. 
Entre os modelos de simples entrada, o de DissescuMeyer apresentou o melhor ajuste, porém com elevada multicolinearidade. O modelo de Kopezki-Gherardt também estimou os volumes com boa precisão, havendo sobreposição nas curvas com os valores estimados com os dois modelos (Figura 1C). Sugere-se que esta equação pode ser empregada quando não for possível medir a altura comercial ou quando este dado não estiver disponível.

Apesar de estimar o volume com menor precisão do que a equação de Schumacher e Hall, o emprego desta equação explicou $84 \%$ da variação em volume. Devese salientar que a determinação da altura comercial em florestas nativas é uma operação demorada, trabalhosa e sujeita a erros que, conforme Soares et al. (2006), podem estar relacionado ao objeto medido, aos instrumentos e ao observador. Estes erros não amostrais podem reduzir o ganho de precisão obtido com modelos de dupla entrada.

Ao compararmos os dois modelos selecionados com as estimativas obtidas pela fórmula alemã empregandose $\mathrm{f}=0,7$, obteve-se um erro de estimativa médio de $-0,020 \pm 0,675 \mathrm{~m}^{3}$ por árvore pelo emprego da equação de Schumacher e Hall, 0,003 $\pm 1,267 \mathrm{~m}^{3}$ para a equação de Dissescu-Meyer e -0,989 $\pm 0,760 \mathrm{~m}^{3}$ pelo emprego da fórmula alemã, indicando a baixa precisão deste último método para a floresta estudada.

Os maiores erros ocorreram na determinação do volume comercial individual com casca de Ocotea caudata com -1,977 $\mathrm{m}^{3},-2,443 \mathrm{~m}^{3}$ e $-3,391 \mathrm{~m}^{3}$, respectivamente, que foi a espécie que apresentou maior variabilidade de volume comercial.

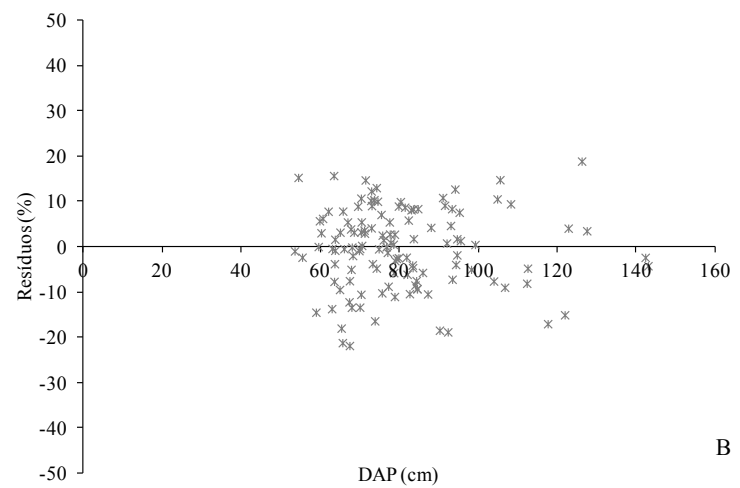

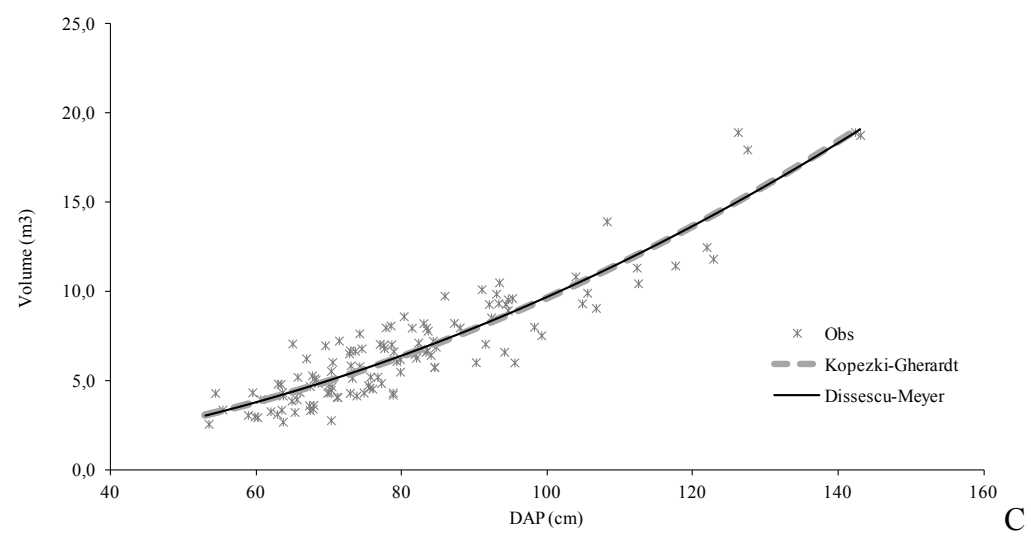

Figura 2. Distribuição dos resíduos para os modelos de Spurr (A), Schumacher e Hall (B) e valores observados e estimados pelos modelos de Dissescu-Meyer e Kopezki-Gherardt (C). 


\section{Conclusões}

Para a estimativa do volume de madeira em Floresta Ombrófila Densa de Terra Firme, no município de Caracaraí, recomenda-se o emprego do modelo individual de Schumacher e Hall, podendo o volume comercial com casca ser estimado pela equação $v=\exp (-8,89142+1,937052 \ln d a p+0,824841 \ln h c$. O modelo de Kopezki-Gherardt $v=0,51168+0,000911$ dap $^{2}$ pode ser empregado quando não se utilizar dados de altura comercial.

\section{Referências}

BARBOSA, R. I. Distribuiçao das chuvas em Roraima. In: BARBOSA, R. I; FERREIRA, E. J. G;CASTELLÓN, E. G. (Ed.). Homem, ambiente e ecologia no estado de Roraima. Manaus: INPA, 1997, p. 325-335.

BARROS, P. L. C. de; SILVA JÚNIOR, A. T. da. Equação de volume para árvores de uma floresta tropical densa no município de Anapu, oeste do Estado do Pará, Amazônia Oriental. Revista de Ciências Agrárias, Belém, n. 51, p. 115-126, 2009.

BARTOSZECK, A. C. de P. e S.; MACHADO, S. do A.; FIQUEIREDO FILHO, A.; OLIVEIRA, E. B. de. Modelagem da relação hipsométrica para bracatingais da região metropolitana de Curitiba-PR. Floresta, Curitiba, v. 32, n. 2, p. 189-204, 2002. DOI: 10.5380/rf.v32i2.2285

BRASIL. Ministério do Meio Ambiente. IBAMA. Instrução normativa $n^{0} 30$, de 31 de dezembro de 2002. Diário Oficial [da] República Federativa do Brasil, Brasília, DF, 1 jan. 2003.

COLPINI, C.; TRAVAGIN, D. P.; SOARES, T. S.; SILVA, V. S. M. e. Determinação do volume, fator de forma e da porcentagem de casca de árvores individuais em uma Floresta Ombrófila Aberta na região noroeste de Mato Grosso. Acta Amazônica, Manaus, v. 39, n. 1. p. 97-104, 2009. DOI: 10.1590/S0044-5967200900100010

CONDE, T. M.; TONINI, H. Fitossociologia de uma Floresta Ombrófila Densa na Amazônia Setentrional, Roraima, Brasil. Acta Amazonica, Manaus, v. 43, n. 3, p. 247-260, 2013.
DRAPER, N.; SMITH, H. Applied regression analysis. $3 \mathrm{rd} \mathrm{ed.}$ New York: Wiley, 1998. 736 p.

FERNANDES, N. P.; JARDIM, F. C.; HIGUCHI, N. Tabelas de volume para a floresta de terra firme da estação experimental de silvicultura tropical. Acta Amazonica, Manaus, v. 13, n. 3-4, p. 537-545, 1983.

FINGER, C. A. G. Fundamentos de biometria florestal. Santa Maria, RS: UFSM, 1992. 269 p.

HEINSDJIK, D.; BASTOS, A. M. Inventários florestais na Amazônia. Rio de Janeiro: Ministério da Agricultura, 1963. 100 p. (Boletim, 6).

HIGUCHI, N.; RAMM, W. Developing bole wood volume equations for a group of tree species of central Amazon (Brazil). The Commonwealth Forestry Review, v. 64, n. 1, p. 33-41, 1995.

MACHADO, S. A.; FIGUEIREDO FILHO, S. A. Dendrometria. Curitiba, 2003. 309 p.

NOGUEIRA, E. M.; FEARNSIDE, P. M.; NELSON, B. W.; BARBOSA, R. I.; KEIZER, E. W. H. Estimates of forest biomass in the Brazilian Amazon: new alometric equations and adjustments to biomass from wood-volume inventories. Forest Ecology and Management, v. 256, p. 1853-1867, 2008. 10.1016/j. foreco.2008.07.022

ROLIM, S. G.; COUTO, H. T. Z.; JESUS, R. M.; FRANÇA, J. T. Modelos volumétricos para a Floresta Nacional do Taipé-Aquirí, Serra dos Carajás. Acta Amazonica, Manaus, v. 36, n. 1, p. 106-114, 2006. DOI: $10.1590 / \mathrm{S} 0044-59672006000100013$

SILVA, J. N. M.; ARAUJO, S. M. Equação de volume para árvores de pequeno diâmetro na Floresta Nacional do Tapajós. Boletim de Pesquisa Florestal, Colombo, n. 8/9, p.16-25, 1984.

SOARES, C. P. B.; NETO, F. P; SOUZA, A. L. Dendrometria e inventário florestal. Viçosa, MG: Ed da UFV, 2006. 276 p.

THAINES, F.; BRAZ, E. M.; MATTOS, P. P.; THAINES, A. A. R. Equações para a estimative de volume de madeira para a região da bacia do Rio Ituxi, Lábrea, AM. Pesquisa Florestal Brasileira, Colombo, v. 30, n. 64, p. 283-289, 2010. DOI: 10.4336/2010. pfb.30.64.283

VALE VERDE . Madeireira Vale Verde Ltda. Disponivel em : $<$ www. madeireira-vale-verde.com>. Acesso em 07 de fevereiro de 2012. 
